\title{
A Benchmark Problem for Robust Control of a Multivariable Nonlinear Flexible Manipulator
}

Stig Moberg, Jonas Öhr, Svante Gunnarsson

Division of Automatic Control

E-mail: stig@isy.liu.se, jonas.ohr@optimation.se, svante@isy.liu.se

17th March 2008

Report no.: LiTH-ISY-R-2848

Accepted for publication in 17th IFAC World Congress, Seoul, Korea

Address:

Department of Electrical Engineering

Linköpings universitet

SE-581 83 Linköping, Sweden

WWW: http://www.control.isy.liu.se

AUTOMATIC CONTROL

REGLERTEKNIK

LINKÖPINGS UNIVERSITET

Technical reports from the Automatic Control group in Linköping are available from

http://www.control.isy.liu.se/publications. 


\begin{abstract}
A benchmark problem for robust feedback control of a manipulator is presented. The system to be controlled is an uncertain nonlinear two link manipulator with elastic gear transmissions. The gear transmission is described by nonlinear friction and elasticity. The system is uncertain according to a parametric uncertainty description and due to uncertain disturbances affecting both the motors and the tool. The system should be controlled by a discrete-time controller that optimizes performance for given robustness requirements. The control problem concerns only disturbance rejection. The proposed model is validated by experiments on a real industrial manipulator.
\end{abstract}

Keywords: Robust control, control, benchmark examples, manipulators, disturbance rejection, flexible arms, robotics 


\title{
A Benchmark Problem for Robust Control of a Multivariable Nonlinear Flexible Manipulator
}

\author{
Stig Moberg ${ }^{*, * *}$ Jonas Öhr ${ }^{* *}$ Svante Gunnarsson ${ }^{* * *}$ \\ * ABB AB - Robotics, SE-721 68 Västerås, Sweden, \\ (e-mail: stig.moberg@se.abb.com) \\ ** Optimation, SE-753 20 Uppsala, Sweden \\ *** Division of Automatic Control, Department of Electrical \\ Engineering, Linköping University, SE-581 83 Linköping, Sweden
}

\begin{abstract}
A benchmark problem for robust feedback control of a manipulator is presented. The system to be controlled is an uncertain nonlinear two link manipulator with elastic gear transmissions. The gear transmission is described by nonlinear friction and elasticity. The system is uncertain according to a parametric uncertainty description and due to uncertain disturbances affecting both the motors and the tool. The system should be controlled by a discrete-time controller that optimizes performance for given robustness requirements. The control problem concerns only disturbance rejection. The proposed model is validated by experiments on a real industrial manipulator.
\end{abstract}

Keywords: Robust control, control, benchmark examples, manipulators, disturbance rejection, flexible arms, robotics

\section{INTRODUCTION}

There exists a gap between control theory and control practise, i.e., many control methods suggested by researchers are seldom implemented in real systems and, on the other hand, many important industrial control problems are not studied in the academic research. This is recognized in, e.g. Åström (1994) where the need for a balance between theory and practise is expressed. From Bernstein (1999) we quote "I personally believe that the gap on the whole is large and warrants serious introspection by the research community". The same article also points out that the control practitioners must articulate their needs to the research community, and that motivating the researchers with problems from real applications "can have a significant impact on increasing the relevance of academic research to engineering practise".

This paper presents an industrial benchmark problem with the intention to stimulate research in the area of robust control of flexible industrial manipulators and thus bridging the gap between control theory and practice. The MIMO benchmark problem presented in this article is an extension of a similar SISO problem presented in Moberg and Öhr (2005). The SISO benchmark model is experimentally validated and further described together with an analysis of some suggested solutions in Moberg et al. (2007). In summary, the SISO problem can be solved with a PID controller and it is in fact hard to improve the performance further no matter which controller is used. This is quite a surprising result and now the investigation continues. The main difference of the new problem is that the realism is increased one step further, not only by making the problem multivariable, but also by adding some nonlinearities ignored in the previous benchmark.

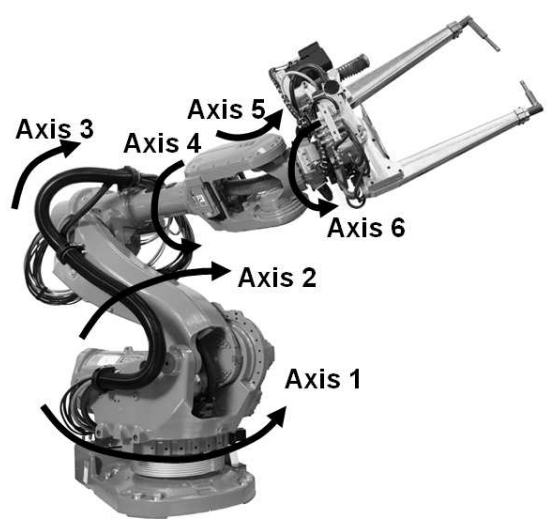

Fig. 1. IRB6600 from ABB equipped with a spot welding gun

The paper is organized as follows. Section 2 presents the control problem, and Section 3 presents the nonlinear manipulator model. Section 4 describes the complete benchmark system, and the proposed model structure is validated by experiments on a real industrial manipulator in Section 5. Finally, the control design task is presented in Section 6.

\section{PROBLEM DESCRIPTION}

The most common type of industrial manipulator has six serially mounted links, all controlled by electrical motors via gears. An example of a serial industrial manipulator is shown in Figure 1. The dynamics of the manipulator change rapidly when the robot links move fast within the manipulator workspace, and the dynamic couplings between the links are in general strong. Moreover, the structure is elastic and the gears have nonlinearities such 
as hysteresis, backlash, friction and nonlinear elasticity. From a control engineering perspective a manipulator can be described as a nonlinear multivariable dynamical system having the six motor currents as the inputs and the six motor angles as measurable outputs. The main objective of the motion control is, however, to control the orientation and the position of the tool when moving the tool along a certain desired path.

The benchmark problem described in this paper concerns only the so-called regulator problem, where a feedback controller should be designed such that the tool position is close to the desired reference, in the presence of motor torque disturbances, e.g., motor torque ripple, and force disturbances acting on the tool, e.g., during material processing. Only the second and third links of the manipulator will be included in the benchmark model. These links are chosen in order to get a strong dynamic coupling. The model will include the nonlinear rigid body dynamics associated with the change of configuration (link positions) as well as gravity, centripetal and Coriolis torques. Moreover, the nonlinear elasticity and friction of the gear transmissions will be included in the model.

The rationale behind the different choices in the problem design is that a benchmark problem should be sufficiently realistic and complete to act as a substitute for real control experiments. However, the number of researchers who will have time and resources to take on the problem and propose solutions and methods, will certainly decrease with increased problem complexity. If reference tracking was included in the problem specification and/or if all links of an industrial manipulator were included in the model, both the realism and the complexity of the problem would increase. The suggested benchmark problem is hopefully a good trade-off.

\section{THE MANIPULATOR MODEL}

The two link manipulator is a model of link 2 and 3 for a typical large industrial robot, see Figure 1 . The model is planar, i.e., all movements are constrained to the $\mathrm{x}, \mathrm{z}$ plane. The model is illustrated in Figure 2. In the following, the links are denoted as link 1 and link 2. Each link has the following rigid body attributes:

- mass $m_{1}$ and $m_{2}$

- link length $l_{1}$ and $l_{2}$

- center of mass $\xi_{1}$ and $\xi_{2}$ (distances from the centers of rotation)

- inertia w.r.t. center of mass $j_{1}$ and $j_{2}$

The links are actuated by electrical motors, connected to the links via elastic joints. The joints (gear transmissions) are described by the nonlinear spring torque $\tau_{s}(q)$, the linear damping matrix $D$, the friction torque $f(\dot{q})$, and the gear ratios $\left(n_{1}\right.$ and $\left.n_{2}\right)$. The motors are described by their inertias $j_{m 1}$ and $j_{m 2}$. There are two degrees of freedom (DOF) for each axis described by the motor angular positions $q_{m 1}, q_{m 2}$ and link angular positions $q_{a 1}$, $q_{a 2}$. The control signals are the motor torques $u_{m 1}$ and $u_{m 2}$, which are subject to saturation. The motor torque control is modeled as a gain uncertainty $\gamma$ and a time delay $T_{d 1}$. The only measured output signals are the motor angular positions, and these are subject to measurement noise and a time delay $T_{d 2}$. This time delay is motivated by the computational and communication delay. Two sources of disturbance are acting on the system. A force $\mathrm{F}$ is applied at the tool center point (TCP) at angle $\phi_{F}$ and a

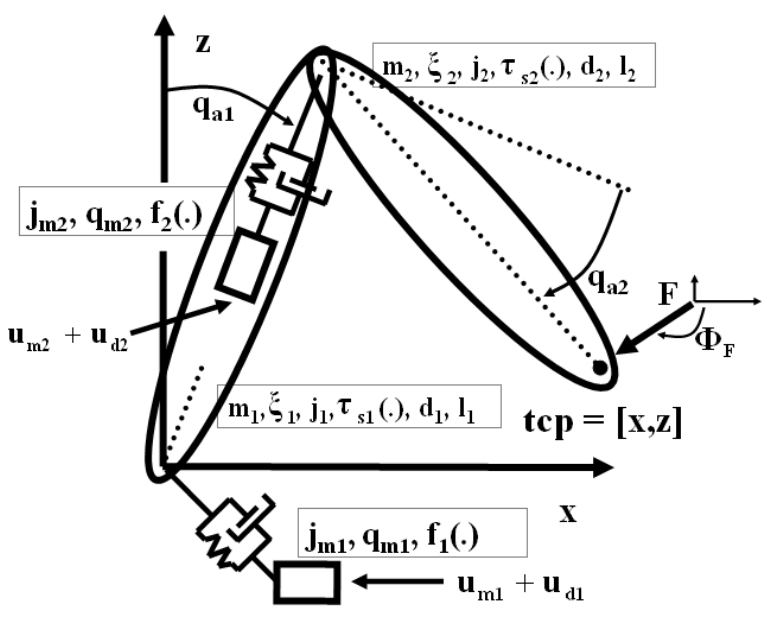

Fig. 2. A two link robot model

motor torque disturbance is applied as input disturbance signals $u_{d 1}$ and $u_{d 2}$. The angular positions and the model inputs are described by

$$
q=\left[\begin{array}{c}
q_{a 1} \\
q_{a 2} \\
q_{m 1} / n_{1} \\
q_{m 2} / n_{2}
\end{array}\right], \quad u=\left[\begin{array}{c}
u_{a 1} \\
u_{a 2} \\
\left(u_{m 1}+u_{d 1}\right) n_{1} \\
\left(u_{m 2}+u_{d 2}\right) n_{2}
\end{array}\right] .
$$

The manipulator is described by its dynamics

$$
u=M(q) \ddot{q}+C(q, \dot{q})+G(q)+D \dot{q}+\tau_{s}(q)+f(\dot{q}) .
$$

The inertial coupling between the motor and link rotation is neglected due to the high gear ratio, see e.g. Spong (1987). The inertia matrix $M$, gravity vector $G$ and vector of speed dependent torques (Coriolis and centripetal) $C$ can then easily be derived as (see e.g. Sciavicco and Siciliano (2000))

$$
\begin{aligned}
& M(q)=\left[\begin{array}{cccc}
J_{11}(q) & J_{12}(q) & 0 & 0 \\
J_{21}(q) & J_{22}(q) & 0 & 0 \\
0 & 0 & j_{m 1} n_{1}^{2} & 0 \\
0 & 0 & 0 & j_{m 2} n_{2}^{2}
\end{array}\right], \\
& J_{11}(q)=j_{1}+m_{1} \xi_{1}^{2}+j_{2}+m_{2}\left(l_{1}^{2}+\xi_{2}^{2}-2 l_{1} \xi_{2} \sin q_{a 2}\right) \text {, } \\
& J_{12}(q)=J_{21}(q)=j_{2}+m_{2}\left(\xi_{2}^{2}-l_{1} \xi_{2} \sin q_{a 2}\right), \\
& J_{22}(q)=j_{2}+m_{2} \xi_{2}^{2} \text {, } \\
& G(q)=\left[\begin{array}{llll}
g_{1}(q) & g_{2}(q) & 0 & 0
\end{array}\right]^{T}, \\
& g_{1}(q)=-g\left(m_{1} \xi_{1} \sin \left(q_{a 1}\right)+\right. \\
& \left.m_{2}\left(l_{1} \sin \left(q_{a 1}\right)+\xi_{2} \cos \left(q_{a 1}+q_{a 2}\right)\right)\right), \\
& g_{2}(q)=-m_{2} \xi_{2} g \cos \left(q_{a 1}+q_{a 2}\right) \text {, } \\
& C(q, \dot{q})=\left[\begin{array}{c}
\left.-m_{2} l_{1} \xi_{2} \cos \left(q_{a 2}\right)\left(2 \dot{q}_{a 1} \dot{q}_{a 2}+\dot{q}_{a 2}^{2}\right)\right) \\
m_{2} l_{1} \xi_{2} \cos \left(q_{a 2}\right) \dot{q}_{a 1}^{2} \\
0 \\
0
\end{array}\right],
\end{aligned}
$$

where $g$ is the gravitational constant. The nonlinear spring torque is given by

$$
\begin{aligned}
\tau_{s}(q) & =\left[\begin{array}{c}
\tau_{s 1}\left(\Delta_{q 1}\right) \\
\tau_{s 2}\left(\Delta_{q 2}\right) \\
\tau_{s 1}\left(-\Delta_{q 1}\right) \\
\tau_{s 2}\left(-\Delta_{q 2}\right)
\end{array}\right], \\
\Delta_{q i} & =q_{a i}-q_{m i} / n_{i}, \quad i=1 \ldots 2 .
\end{aligned}
$$

with 


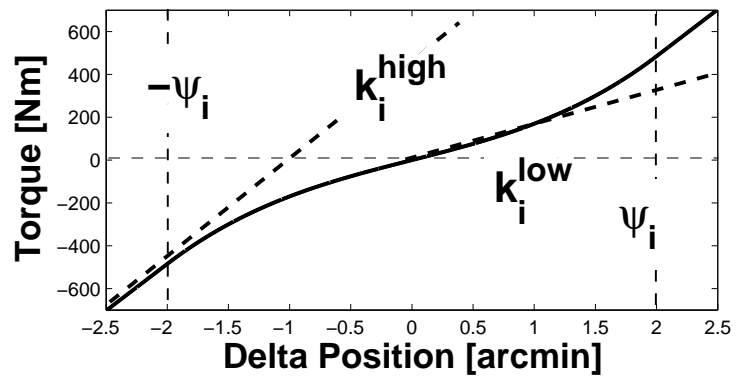

Fig. 3. Example of nonlinear stiffness (elasticity).

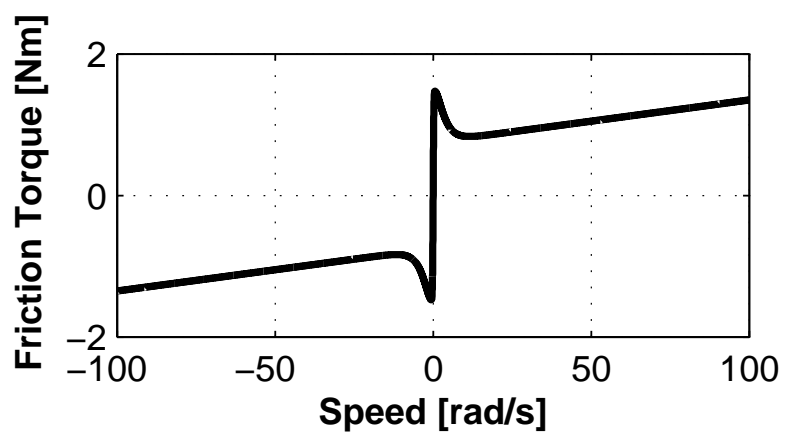

Fig. 4. Example of nonlinear friction.

$$
\begin{aligned}
\tau_{s i} & =k_{i 1} \Delta_{q i}+k_{i 3} \Delta_{q i}^{3},\left|\Delta_{q i}\right| \leq \psi_{i}, \\
\tau_{s i} & =\operatorname{sign}\left(\Delta_{q i}\right)\left(m_{i 0}+m_{i 1}\left(\left|\Delta_{q i}\right|-\right.\right. \\
k_{i 1} & =k_{i}^{\text {low }} \\
k_{i 3} & =\left(k_{i}^{\text {high }}-k_{i}^{\text {low }}\right) /\left(3 \psi_{i}^{2}\right), \\
m_{i 0} & =k_{i 1} \psi_{i}+k_{i 3} \psi_{i}^{3}, \\
m_{i 1} & =k_{i}^{\text {high }} .
\end{aligned}
$$$$
\tau_{s i}=\operatorname{sign}\left(\Delta_{q i}\right)\left(m_{i 0}+m_{i 1}\left(\left|\Delta_{q i}\right|-\psi_{i}\right)\right),\left|\Delta_{q i}\right|>\psi_{i},
$$

The nonlinear stiffness (elasticity) is then specified by the lowest stiffness $k_{i}^{\text {low }}$, the highest stiffness $k_{i}^{\text {high }}$, and the breakpoint deflection $\psi_{i}$, see Figure 3 . The linear damping matrix is

$$
D=\left[\begin{array}{cccc}
d_{1} & 0 & -d_{1} & 0 \\
0 & d_{2} & 0 & -d_{2} \\
-d_{1} & 0 & d_{1} & 0 \\
0 & -d_{2} & 0 & d_{2}
\end{array}\right]
$$

The nonlinear friction torque, see Figure 4, is approximated as acting on the motor only and is given by the following equation

where

$$
f(\dot{q})=\left[\begin{array}{llll}
0 & 0 & f_{1}(\dot{q}) & f_{2}(\dot{q})
\end{array}\right]^{T},
$$

$$
\begin{aligned}
f_{i}(\dot{q})= & n_{i}\left(f_{d i} \dot{q}_{m i}+f_{c i}\left(\mu_{k i}+\left(1-\mu_{k i}\right)\right.\right. \\
& \left.\left.\cosh ^{-1}\left(\beta_{i} \dot{q}_{m i}\right)\right) \tanh \left(\alpha_{i} \dot{q}_{m i}\right)\right), \quad i=1 \ldots 2 .
\end{aligned}
$$

This smooth friction model is suggested in Feeny and Moon (1994) and avoids discontinuities to simplify numerical integration. The TCP position $X$ is described by the kinematics

$$
X=\Gamma(q)=\left[\begin{array}{l}
x(q) \\
z(q)
\end{array}\right]=\left[\begin{array}{l}
l_{1} \sin \left(q_{a 1}\right)+l_{2} \cos \left(q_{a 1}+q_{a 2}\right) \\
l_{1} \cos \left(q_{a 1}\right)-l_{2} \sin \left(q_{a 1}+q_{a 2}\right)
\end{array}\right]
$$

The relation between the disturbance force $F$ and joint torques $u_{a}$ is given by the velocity Jacobian $J\left(q_{a}\right)$ as

$$
u_{a}=J^{T}\left(q_{a}\right) F, \quad u_{a}=\left[\begin{array}{l}
u_{a 1} \\
u_{a 2}
\end{array}\right], \quad F=\left[\begin{array}{c}
F \cos \left(\phi_{F}\right) \\
F \sin \left(\phi_{F}\right)
\end{array}\right],
$$

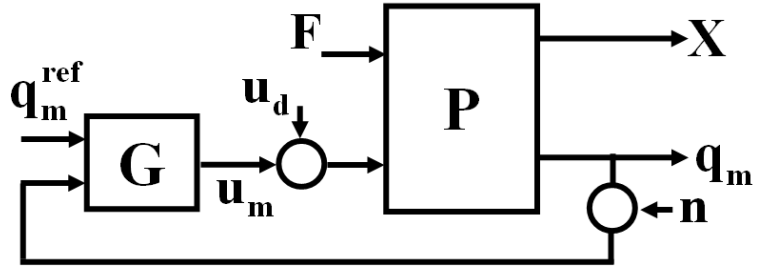

Fig. 5. A block diagram of the benchmark system

$$
\begin{aligned}
& J\left(q_{a}\right)=\left[\frac{\partial \Gamma\left(q_{a}\right)}{\partial q_{a}}\right] \\
= & {\left[\begin{array}{cc}
l_{1} \cos \left(q_{a 1}\right)-l_{2} \sin \left(q_{a 1}+q_{a 2}\right) & -l_{2} \sin \left(q_{a 1}+q_{a 2}\right) \\
-l_{1} \sin \left(q_{a 1}\right)-l_{2} \cos \left(q_{a 1}+q_{a 2}\right) & -l_{2} \cos \left(q_{a 1}+q_{a 2}\right)
\end{array}\right] . }
\end{aligned}
$$

\section{THE BENCHMARK SYSTEM}

The benchmark system consists of the manipulator model $P$ described in Section 3 and a feedback controller $G$ as illustrated in Figure 5. The model uncertainty description is parametric and expressed as uncertainty in some of the physical parameters. The friction and stiffness uncertainties are motivated by modeling errors and differences between the gearbox individuals. The mass uncertainty is due to errors in the definition of the user loads attached to the manipulator and the gain error reflects to the accuracy of the torque control.

The discrete-time controller $G$ is implemented with sample time $T_{s}$, time delay $T_{d}$ and a control signal limitation $u_{m}^{\max }$. The time delay includes both the delay of the torque control and the computational and communication delay described in Section 3, i.e., $T_{d}=T_{d 1}+T_{d 2}$. The DA and $\mathrm{AD}$ conversions are modeled by a 12 bit quantization of the output torque and a 16 bit quantization of the motor position.

The system is influenced by the following uncertain disturbances: a measurement noise $n$ with power $P_{n}$, a disturbance force $F$ in direction $\phi_{F}$ applied at $t_{1}$ and released at $t_{2}$ and finally a motor torque disturbance $u_{d}$ applied from $t_{3}$ to $t_{4}$. $F$ can be applied in any direction and the torque disturbance $u_{d}$ is a chirp with amplitude $A_{c}$, start frequency $f_{c s}$ and end frequency $f_{c e}$. The motor torque disturbance is motivated by internally generated ripple disturbances due to the design of the motors and the gear boxes. These disturbances have frequency components proportional to the motor speed and can cause significant position errors in some frequency regions. The force disturbance is motivated by various externally generated disturbances, e.g., the release of a load, forces due to material processing, or forces due to the impact at spotwelding. The force disturbance pulse serves as a generalization of all real application-specific disturbances. The manipulator model parameters, the controller parameters, the disturbance parameters, and the uncertainty descriptions are listed in Table 1. The parameters with no axis index are the same for both axes although the uncertainty is independent for each parameter. The parameter values and the uncertainties are known (by experience) to be realistic, although the exact combination of parameters used do not correspond to a specific industrial robot. 
Table 1. Nominal and Uncertain Parameters

\begin{tabular}{|c|c|c|c|}
\hline Parameter & Value & Unit & Uncertainty \\
\hline$j_{m 1}$ & 0.004 & $\mathrm{~kg} \cdot \mathrm{m}^{2}$ & \\
\hline$j_{m 2}$ & 0.001 & $\mathrm{~kg} \cdot \mathrm{m}^{2}$ & \\
\hline$j_{1}$ & 5 & $\mathrm{~kg} \cdot \mathrm{m}^{2}$ & \\
\hline$j_{2}$ & 50 & $\mathrm{~kg} \cdot \mathrm{m}^{2}$ & \\
\hline$m_{1}$ & 50 & $\mathrm{~kg}$ & $\pm 10 \%$ \\
\hline$m_{2}$ & 150 & $k g$ & $\pm 10 \%$ \\
\hline$l_{1}$ & 1.0 & $m$ & \\
\hline$l_{2}$ & 1.5 & $m$ & \\
\hline$\xi_{1}$ & 0.5 & $m$ & \\
\hline$\xi_{2}$ & 0.8 & $m$ & \\
\hline$n$ & 200 & & \\
\hline$k_{1}^{h i g h}$ & $0.5 \cdot 10^{6}$ & $\mathrm{Nm} / \mathrm{rad}$ & $\pm 20 \%$ \\
\hline$k_{1}^{\text {tow }}$ & $1.5 \cdot 10^{6}$ & $\mathrm{Nm} / \mathrm{rad}$ & $\pm 20 \%$ \\
\hline$\psi_{1}$ & 2 & arcmin & $\pm 20 \%$ \\
\hline$k_{2}^{h i g h}$ & $0.2 \cdot 10^{6}$ & $\mathrm{Nm} / \mathrm{rad}$ & $\pm 20 \%$ \\
\hline$k_{2}^{\text {tow }}$ & $0.6 \cdot 10^{6}$ & $\mathrm{Nm} / \mathrm{rad}$ & $\pm 20 \%$ \\
\hline$\overline{\psi_{2}}$ & 3 & arcmin & $\pm 20 \%$ \\
\hline$d_{1}$ & 600 & $N m \cdot s / r a d$ & $\pm 20 \%$ \\
\hline$d_{2}$ & 200 & $N m \cdot s / r a d$ & $\pm 20 \%$ \\
\hline$f_{v 1}$ & 0.006 & $N m \cdot s / r a d$ & $\pm 80 \%$ \\
\hline$f_{c 1}$ & 1.5 & $N m$ & $\pm 80 \%$ \\
\hline$f_{v 2}$ & 0.003 & $N m \cdot s / r a d$ & $\pm 80 \%$ \\
\hline$f_{c 2}$ & 1.0 & $N m$ & $\pm 80 \%$ \\
\hline$\mu$ & 0.5 & & $\pm 50 \%$ \\
\hline$\beta$ & 0.4 & & $\pm 50 \%$ \\
\hline$\alpha$ & 5 & & \\
\hline$g$ & 9.81 & $\mathrm{~m} / \mathrm{s}^{2}$ & \\
\hline$\gamma$ & 1 & & $\pm 10 \%$ \\
\hline$P_{n}$ & $3 \cdot 10^{-12}$ & & \\
\hline$F$ & 500 & $N$ & \\
\hline$\phi_{F}$ & $\pi$ & rad & $\pm \pi$ \\
\hline$t_{1}$ & 10 & $s$ & \\
\hline$t_{2}$ & 10.5 & $s$ & \\
\hline$A_{c 1}$ & 1 & $N m$ & \\
\hline$A_{c 2}$ & -1 & $N m$ & \\
\hline$t_{3}$ & 0.5 & $s$ & $\pm 0.5 \mathrm{~s}$ \\
\hline$t_{4}$ & 8 & $s$ & \\
\hline$f_{c s}$ & 0 or 15 & $H z$ & random choice \\
\hline$f_{c e}$ & 0 or 15 & $H z$ & random choice \\
\hline$T_{s}$ & $0.25 \cdot 10^{-3}$ & $s$ & \\
\hline$T_{d}$ & $0.25 \cdot 10^{-3}$ & $s$ & \\
\hline$u_{\operatorname{mix}}^{\max }$ & 35 & $\mathrm{Nm}$ & \\
\hline$u_{\max }^{\max }$ & 20 & $\mathrm{Nm}$ & \\
\hline$K_{p}^{m^{2}}$ & 45 & & \\
\hline$K_{i}^{1}$ & 30 & & \\
\hline$K_{d}^{1}$ & 1.5 & & \\
\hline$K_{p}^{a}$ & 15 & & \\
\hline$K_{i}^{2}$ & 10 & & \\
\hline$K_{d}^{2}$ & 0.5 & & \\
\hline$z_{p}$ & 0.95 & & \\
\hline
\end{tabular}

The benchmark system, available for download, has a discrete-time diagonal PID controller with derivative filter described by the transfer function

$$
G_{p i d}(z)=\left[\begin{array}{cc}
g_{11}(z) & 0 \\
0 & g_{22}(z)
\end{array}\right]
$$

where

$$
g_{i i}(z)=K_{p}^{i}+K_{d}^{i} \frac{z-1}{T_{s} z} \frac{\left(1-z_{p}^{i}\right) z}{z-z_{p}^{i}}+\frac{K_{i}^{i} T_{s} z}{z-1} .
$$

The PID controller should only be seen as an example of a controller yielding a stable system and does not represent an optimal design.

\section{MODEL VALIDATION}

In this section the model proposed in Section 3 is validated by experiments made on the second and third links of

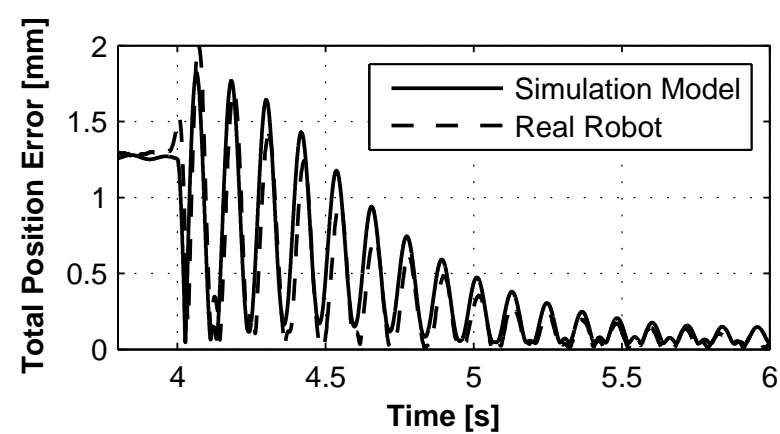

Fig. 6. The tool position error for a tool step force disturbance.

an industrial robot from $\mathrm{ABB}$, using an experimental controller. All model parameters, except the $\alpha$ parameters of the friction model (8) and the damping $D$ in (6), were known with sufficient accuracy. The configuration of the wrist, i.e., axis 4 - 6 , was chosen to minimize the coupling between the modeled links and the wrist. The robot links were controlled with a diagonal PID controller of the same type as the default controller of the benchmark system.

In the first experiment a tool load was instantaneous released, i.e., a step disturbance force was applied. The tool response was measured using a laser measurement system LTD600 described in Leica (2007). The elasticity of the model was then tuned w.r.t. the transient response. The resulting elasticity was somewhat lower than the known elasticity of the gear boxes. This was expected since a modern industrial robot cannot be fully modeled by the so-called flexible joint approach, see, e.g., Moberg and Hanssen (2007). The damping was set to a reasonable value, in fact, the response of the controlled system is quite insensitive to the damping. The remaining unknown model parameter, $\alpha$, was tuned w.r.t. the measured response. Note that the factor $\tanh \left(\alpha \dot{q}_{m}\right)$ in (8) approximates the discontinuous friction behavior at zero speed and cannot be directly measured. The result is shown in Figure 6 . The experiment was repeated for a number of controller tunings with good correspondence between simulation model and real robot.

In the second experiment, a chirp torque disturbance $u_{d}$ was added to the control signal while the manipulator was moving at a low speed. This is motivated by the fact that internally generated torque disturbances are present only when the robot is moving and that the nonlinear friction at zero speed otherwise would reduce the effect of the disturbance, e.g., no movement would be generated if the disturbance level was below the Coulomb friction level. Moving the manipulator at a low speed thus linearizes the system w.r.t. friction and the robot response can thus be compared with the model response when the nonlinear friction, $f_{c}$, is set to zero. This comparison is shown in Figure 7 and the correspondence is good. In the benchmark problem, the chirp disturbance is applied at zero speed. This choice was made to avoid introduction of a reference signal and is justified by the fact that the relative disturbance rejection at zero speed also reflects the disturbance rejection when moving.

The third validation experiment concerned the stability margin of the model. The loop gain of the robot system was increased for one channel at a time until the stability limit was reached. The experimentally determined ampli- 


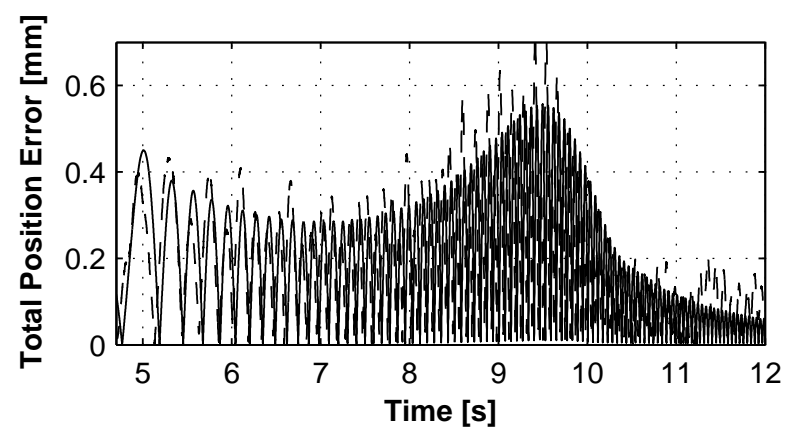

Fig. 7. The tool position error for a motor torque disturbance of chirp type.

tude margin was in good agreement with the one of the simulated system.

\section{THE DESIGN TASK: PERFORMANCE SPECIFICATION AND COST FUNCTION}

Design a discrete-time controller to control the manipulator in the entire manipulator workspace defined by $q_{a 1} \in\left[-90^{\circ} \ldots 180^{\circ}\right]$ and $q_{a 2} \in\left[-180^{\circ} \ldots 80^{\circ}\right]$. The controller can be of any kind, e.g., linear or non-linear, diagonal or full-matrix, time-invariant or gain scheduled. The controller inputs are the measured motor positions and constant motor position references. The motor position references $q_{m}^{r e f}$ and the initial gravity torques may be used for initializing the controller. The motor position given as reference represents a steady-state solution at the desired link position, i.e., the differences between the motor and link initial state is equal to the gravity deflection.

The designed controller should replace the default PID controller but the system described in Section 4 must otherwise be unchanged. For all configurations inside the workspace, for all systems and disturbances in the uncertainty description, the following requirements concerning stability must be fulfilled:

- $A_{1}$ : The system must remain stable for a loop gain increase of a factor of 2.5 (one channel at a time).

- $A_{2}$ : The system must remain stable for a delay increase of $1.5 \mathrm{~ms}$ (one channel at a time).

- $A_{3}$ : Maximum limit cycle peak amplitude in TCP position must be lower than $10 \mu \mathrm{m}$ for all test cases including the stability tests $A_{1}$ and $A_{2}$.

The following requirements are to be regarded as target values for the design:

- $B_{1}$ : Maximum motor torque due to measurement noise axis 1: $0.7 \mathrm{Nm}$

- $B_{2}$ : Maximum motor torque due to measurement noise axis 2: $0.4 \mathrm{Nm}$

- $B_{3}$ : Maximum motor torque axis 1: $35 \mathrm{Nm}$

- $B_{4}$ : Maximum motor torque axis 2: $20 \mathrm{Nm}$

- $B_{5}$ : Max TCP position error due to force disturbance: $7 \mathrm{~mm}$

- $B_{6}$ : Max TCP settling time, i.e., error $<0.1 \mathrm{~mm}$, after end of force disturbance pulse: $3 \mathrm{~s}$

- $B_{7}$ : Max TCP position error due to motor torque disturbance: $0.5 \mathrm{~mm}$

Note that the dynamics of the manipulator varies with the tool position and also due to the uncertainty of the manipulator model. Furthermore, the disturbances are

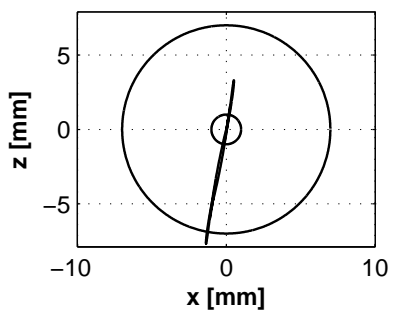

Fig. 8. Target for disturbance rejection w.r.t. tool force disturbance. TCP shall always be inside the large circle, and be inside the small circle after target settling time. Note that the small circle in this figure is enlarged for illustration purposes. The actual radius is $0.1 \mathrm{~mm}$

also uncertain, the force can have any direction and the motor torque ripple can also change direction.

At each configuration $Q_{k}=\left[\begin{array}{ll}q_{a 1} & q_{a 2}\end{array}\right]^{T}$, the following cost function is defined

$$
V_{k}=\sum_{i=1}^{7} w_{i} \max _{\mathcal{P}, \mathcal{D}}\left(b_{i}\right),
$$

where $\mathcal{P}$ is a set containing all manipulator models obtained from the uncertainty description and $\mathcal{D}$ is the corresponding set for the disturbances. The relative fulfillment of specification $B_{i}$ is denoted $b_{i}$, e.g. a settling time of $2 \mathrm{~s}$ gives $b_{6}=2 / 3$. The weights $w_{i}$ are $\left[\begin{array}{lllllll}3 & 3 & 2 & 2 & 25 & 40 & 25\end{array}\right]$, i.e., a controller which fulfils all requirements exactly, has a cost function $V=100$. The design should aim at minimizing the average cost function

$$
V=\frac{1}{N_{Q}} \sum_{k=1}^{N_{Q}} V_{k},
$$

where the performance is evaluated for a suitable grid of $N_{Q}$ configurations in the manipulator work space. The problem of computing the average (w.r.t. workspace) worst case (w.r.t. uncertainty) performance for a non-linear system might seem hard from a theoretical point of view. However, a wisely chosen grid of configurations and a set of assumed worst case uncertainties in combination with some random uncertainties yields a reasonable approximation of the average worst case performance.

The simulation model including the default PID controller is available for download at Moberg (2007) where some additional information about this benchmark problem also can be found. The simulation model is implemented in Matlab ${ }^{\mathrm{TM}}$ and Simulink ${ }^{\mathrm{TM}}$. The approximate average worst case performance for the proposed controller is computed by the model. This computation is based on a predefined set of uncertainties and configurations. Solutions to the problem can be sent to one of the authors for further evaluation. Our plan is that the proposed solutions shall be presented and discussed at, e.g., an invited session at some appropriate future conference.

The target requirements due to force disturbances are illustrated in Figure 8. In Figure 9 - 10, the TCP position errors are shown for the nominal manipulator model controlled by the default PID controller when one example disturbance is applied. The result for 20 uncertain systems, i.e., 20 sets of model and disturbance uncertainties, in one specific position, is shown in Figure 11 - 12. 


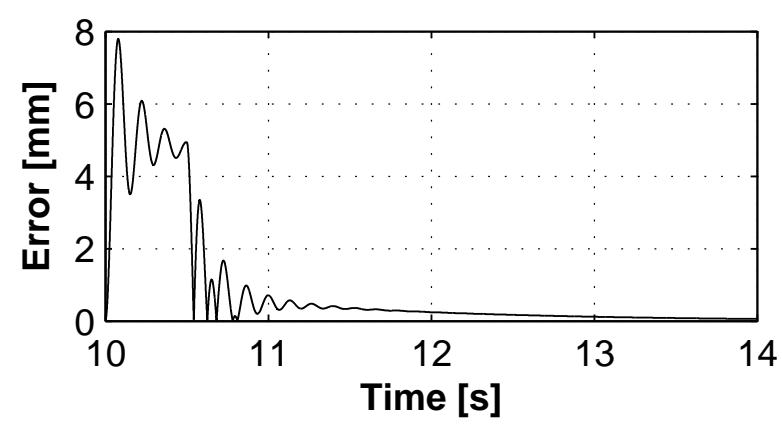

Fig. 9. Example of TCP position error due to force disturbance.

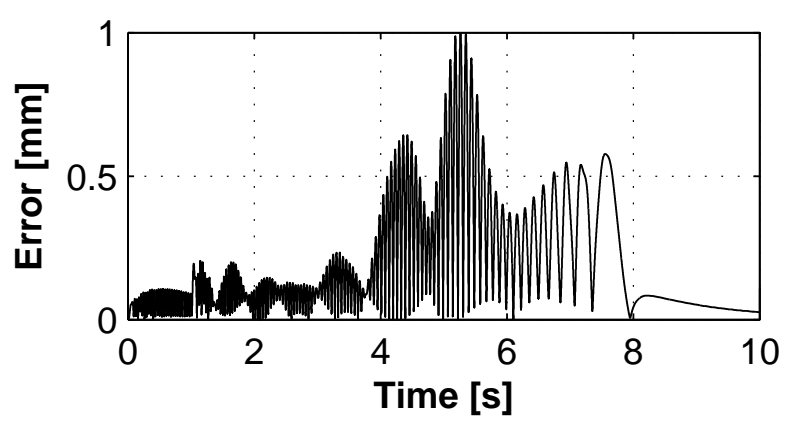

Fig. 10. Example of TCP position error due to motor torque disturbance.

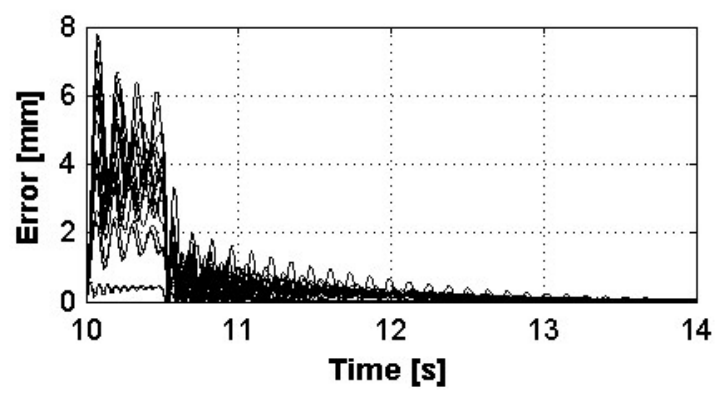

Fig. 11. Example of TCP position error for uncertain system due to force disturbance.

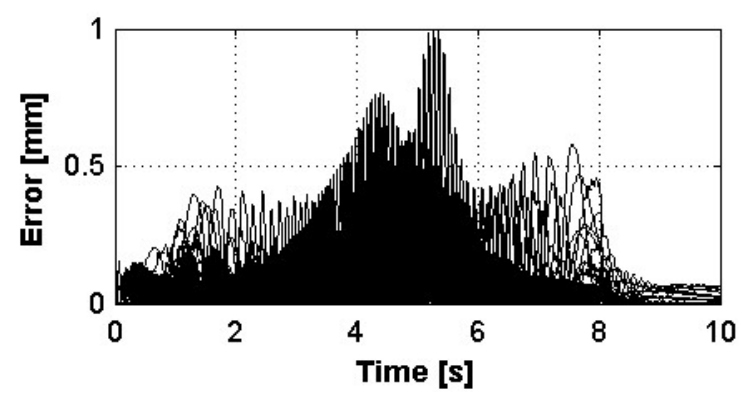

Fig. 12. Example of TCP position error for uncertain system due to motor torque disturbance.

As an example of computation of the average worst case performance, the benchmark system, controlled by the default PID controller, was simulated over a grid of 18 configurations. At each configuration, 3 uncertain systems were evaluated. The target values concerning disturbance rejection, $B_{5}-B_{7}$, are in general not fulfilled. The performance is summarized in Table 2.
Table 2. Average worst case performance of default PID controller

\begin{tabular}{|l|l|l|}
\hline \hline Item & Result for PID & Desired Value \\
\hline$B_{1}$ & 0.17 & 0.7 \\
\hline$B_{2}$ & 0.06 & 0.4 \\
\hline$B_{3}$ & 9.6 & 35 \\
\hline$B_{4}$ & 9.1 & 20 \\
\hline$B_{5}$ & 10.2 & 7 \\
\hline$B_{6}$ & 3.5 & 3 \\
\hline$B_{7}$ & 1.1 & 0.5 \\
\hline$V$ & 141 & 100 \\
\hline
\end{tabular}

7. SUMMARY

A benchmark problem treating disturbance rejection for a nonlinear flexible two-link manipulator has been presented. The system is uncertain due to a parametric uncertainty description and uncertain disturbances affecting both the motors and the tool. The proposed model was validated on a real industrial manipulator. The system should be controlled by a discrete-time controller that optimizes performance for given robustness requirements. Our ambition and hope is that some researchers will be stimulated to work with this benchmark problem using their favorite controller design method. The proposed solutions will be presented at some appropriate future event.

\section{ACKNOWLEDGEMENTS}

The authors would like to thank Sven Hanssen and Sören Quick at ABB Robotics for valuable help and support.

\section{REFERENCES}

D. Bernstein. On bridging the theory/practise gap. IEEE Control Systems Magazine, 19(6):64-70, 1999.

B. Feeny and F. Moon. Chaos in a forced dry-friction oscillator: Experiments and numerical modelling. Journal of Sound and Vibration, 170(3):303-323, 1994.

Leica. Leica geosystems laser trackers. wWw.leica-geosystems.com, 2007.

S. Moberg. Robust control of a multivariable nonlinear flexible manipulator - a benchmark problem. www.robustcontrol.org, 2007.

S. Moberg and S. Hanssen. A dae approach to feedforward control of flexible manipulators. In Proceedings of the IEEE International Conference of Robotics and Automation, April 2007.

S. Moberg and J. Öhr. Robust control of a flexible manipulator arm: A benchmark problem. 16th IFAC World Congress, 2005.

S. Moberg, J. Öhr, and S. Gunnarsson. A benchmark problem for robust feedback control of a flexible manipulator. Technical Report LiTH-ISY-R-2820, Department of Electrical Engineering, Linköpings Universitet, 2007.

L. Sciavicco and B. Siciliano. Modelling and Control of Robot Manipulators. Springer, 2000.

M. W. Spong. Modeling and control of elastic joint robots. Journal of Dynamic Systems, Measurement, and Control, 109:310-319, December 1987.

K. Åström. The future of control. Modeling, Identification and Control, 15(3):127-134, 1994. 


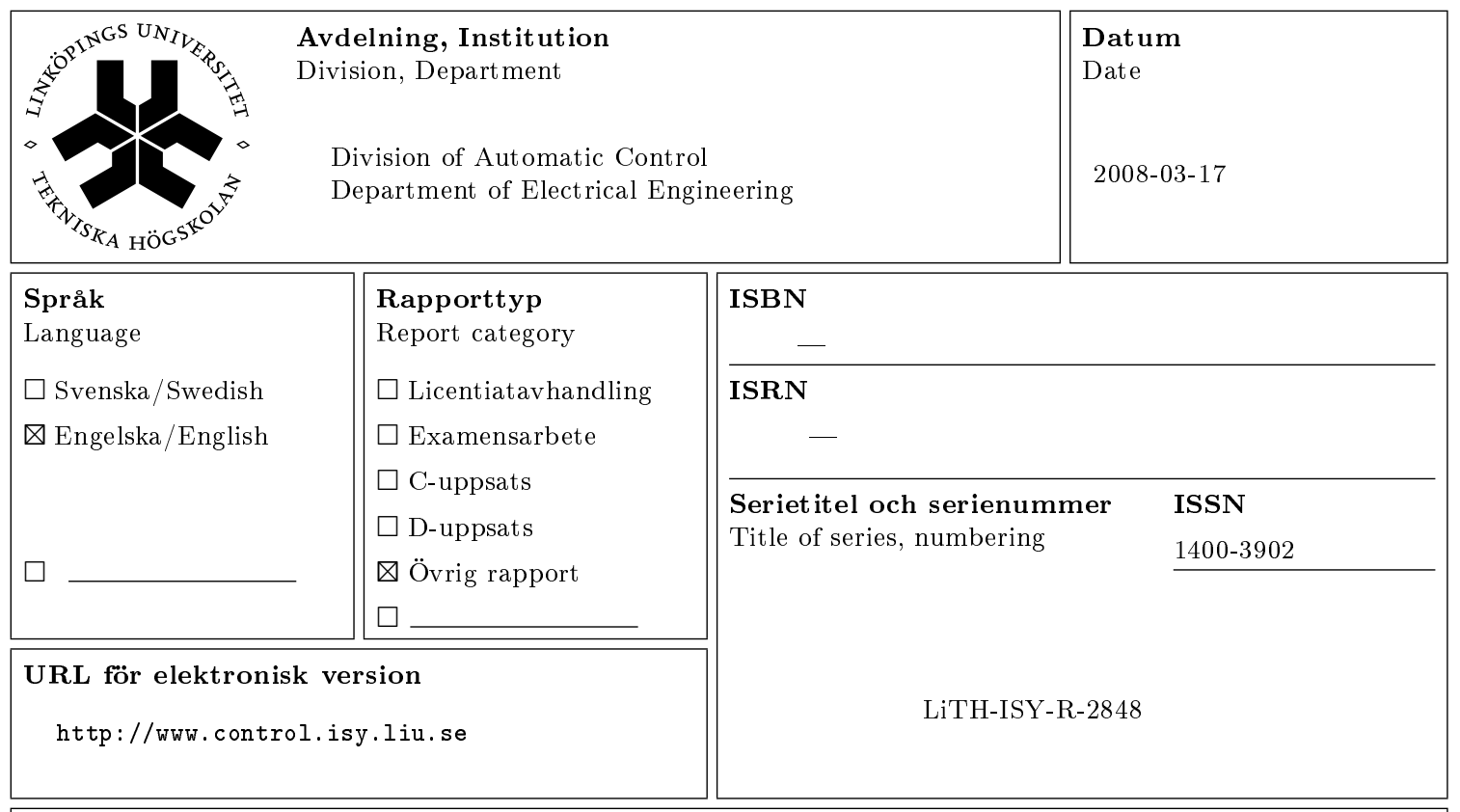

Titel A Benchmark Problem for Robust Control of a Multivariable Nonlinear Flexible Manipulator

Title

Benchmark Problem for Robust Control of a Multivariable Nonlinear Flexible Manipulator

Författare Stig Moberg, Jonas Öhr, Svante Gunnarsson

Author

Sammanfattning

Abstract

A benchmark problem for robust feedback control of a manipulator is presented. The system to be controlled is an uncertain nonlinear two link manipulator with elastic gear transmissions. The gear transmission is described by nonlinear friction and elasticity. The system is uncertain according to a parametric uncertainty description and due to uncertain disturbances affecting both the motors and the tool. The system should be controlled by a discrete-time controller that optimizes performance for given robustness requirements. The control problem concerns only disturbance rejection. The proposed model is validated by experiments on a real industrial manipulator. 